\title{
Management of Mandibular Canine with Accessory Root - A Case Report
}

\author{
Karanam Apoorva Prakash ${ }^{1}$, Shashikala $\mathrm{K}^{2}$, Sunil Mankar ${ }^{3}$, Vanamala $\mathrm{N}^{4}$ \\ ${ }^{1}$ Post Graduate Student, ${ }^{2}$ Professor and Vice Principal, ${ }^{3,4}$ Reader Department Of Conservative Dentistry And \\ Endodontics, D.A.P.M.R.V Dental College, Bangalore
}

\begin{abstract}
Mandibular canine usually present with a single root and root canal. But there are many cases of mandibular canines reported with single root and two root canals, three root canals or with two roots. The aberrant morphology of the mandibular canines should always be considered, because failure to detect the complexities of the internal anatomy results in failure of the endodontic treatment. This clinical case report illustrates the endodontic management of mandibular canine with two roots.
\end{abstract}

Keywords: Endodontic treatment, Mandibular canine, Two roots.

\section{Introduction}

It is of paramount significance that all canals be located and treated throughout the course of nonsurgical endodontic therapy. Teeth with canal bifurcation in the middle or apical third may present problems in the treatment. Usually the canal that is most continuous with the large main passage is acquiscent to sufficient instrumentation and filling procedures, whereas the preparation and filling of the other canal is a great challenge. The incidence of an unfilled canal might elucidate some of the endodontic failures, even though radiographically and clinically the canal appears to be obturated. ${ }^{1}$ This clinical case report illustrates the endodontic management of lower canine with two roots and root canals..

\section{Case Report}

A 43 year old male patient reported with a chief complaint of pain in left lower tooth region since 1 week. Intra oral examination revealed a carious lesion in the left mandibular canine(33) and the tooth was tender to percussion. Vitality tests elicited an abnormal response. Preoperative radiograph showed radiolucency involving enamel, dentin and pulp; with two roots and root canals (figure 1).Presence of two roots was confirmed using CBCT(figure $2 \& 3$ ). A diagnosis of chronic irreversible pulpitis was established and a non surgical endodontic treatment with 33 was planned. Written consent was taken and administration of local anesthesia was done. Caries excavation was carried out and a access was gained into the pulp chamber under rubber dam isolation and a conventional lingual access opening was modified to include more of the incisal surface. The labial and lingual orifices were located with \#10 K-File and were enlarged using Gates-Glidden drills to get a straight line access. Working length determination was done radiographically using Grossmans formula (figure 4). The canals were enlarged by step back preparation using K-Files( Maillefer, Dentsply, Switzerland) and a apical preparation uptill \#40 K-File was done. The root canals were alternatively irrigated with 17\% EDTA and 3\% sodium hypochlorite at every change of instrument and a final irrigation with chlorhexidine was done. An inter appointment calcium hydroxide intra canal medicament was placed and patient was recalled after seven days. In the next visit tooth was found asymptomatic and the canals were obturated using gutta percha by lateral condensation technique (figure $5 \& 6$ ). Post endodontic restoration was done with high strength Glass Ionomer Cement

\section{Discussion}

According to Pecora etal, the internal anatomy, direction and number of roots of 830 extracted human mandibular canines showed presence of a single root in $98.3 \%$ samples, two canals and foramina in $1.2 \%$ teeth and only $1.7 \%$ of the canines had two roots and two root canals. ${ }^{2,3 .}$ D'Arcangelo etal presented two cases of mandibular canines with two roots ${ }^{4}$ Mandibular canine with two roots and three root canals was presented by Heling etal ${ }^{5}$

Multiple roots, root canals and anatomical variations can be identified with the help of a diagnostic radiographs taken in different angulations. Bifurcations in the cervical and middle thirds may be seen radiographically when the X-ray incidence angle does not cause super imposition of images. ${ }^{6}$

appropriate magnification and illumination as the key diagnostic tools for locating canal orifices ${ }^{7}$. Mukhaimer etal suggested staining the pulpal floor with opthalmic dyes, performing champagne or bubble test as the other diagnostic aids for canal location. ${ }^{2}$ A modified wide incisal opening was made instead of a 
traditional lingual entry, as a proper access cavity preparation is vital for successful identification, instrumentation of extra root canals and their obturation. ${ }^{2}$

The more apically the root canal divides, the more difficult it is to access and obturate efficiently. Small precurved K-files are initially used as they can deviate buccally or lingually as the main canal divides, providing a good tactile sensation. ${ }^{8}$ The root canals in mandibular canine are more flattened and less round, but quite large and does not cause technical problems during instrumentation. Two rooted mandibular canines poses a great difficulty in cleaning and shaping. The long axis of canal meets the crown surface at the incisal edge or the labial surface. If this is not taken into consideration, it may lead to deviated preparation ${ }^{9}$

Holtzman etal recommended the use of calcium hydroxide as an inter-visit, intra-canal medicament as instrumentation of such complex root canals is limited, and efficient debridement might be compromised as the anastomoses may connect between the root canals which act as a source of irritants. ${ }^{10}$ Sjogren etal suggested use of calcium hydroxide as an intracanal medicament for a period of 7 days which efficiently eliminates bacteria which may survive biomechanical instrumentation. ${ }^{11}$

Obturation using lateral condensation is considered as gold standard among various other techniques and was used in this case. Mohammadi etal also suggested hybrid technique to fill two separate root canals, as vertical compaction creates an effective apical plug and excellent adaptation to apical gutta percha and to the root canals is achieved by back filling. ${ }^{12}$

Eliyas etal suggested crowns on anterior teeth are best avoided as they experience occlusal forces with lateral vectors during function to protect posterior teeth in guidance. The cervical band of tooth structure especially on the lingual aspect distributes lateral loads and crown preparations on anterior teeth reduces the thickness of this band which results in a reduction in the capability of the tooth to withstand lateral forces thereby causing fracture of the coronal portion at gingival level ${ }^{13}$. Glass ionomer cement was used as a core material because of its cariostatic effect resulting from fluoride release. ${ }^{14}$

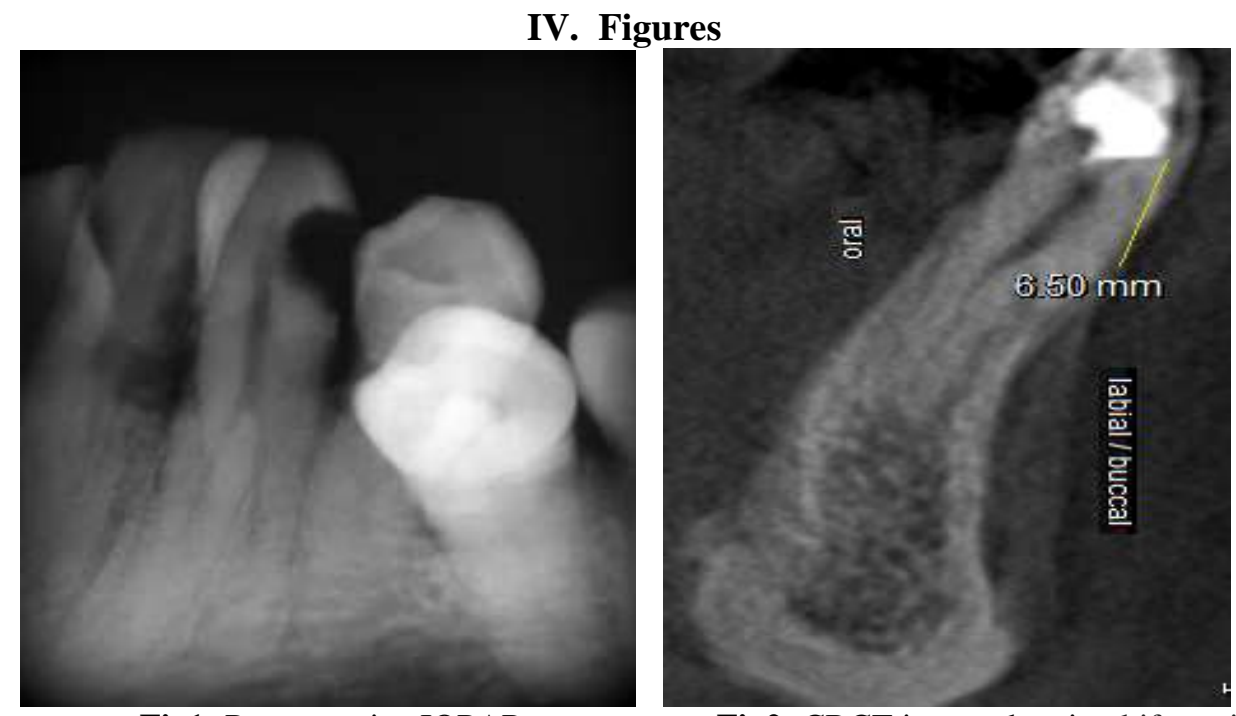

Fig1: Pre-operative IOPAR

Fig2: CBCT image showing bifurcation present approximately at $7 \mathrm{~mm}$ from pulpal floor.

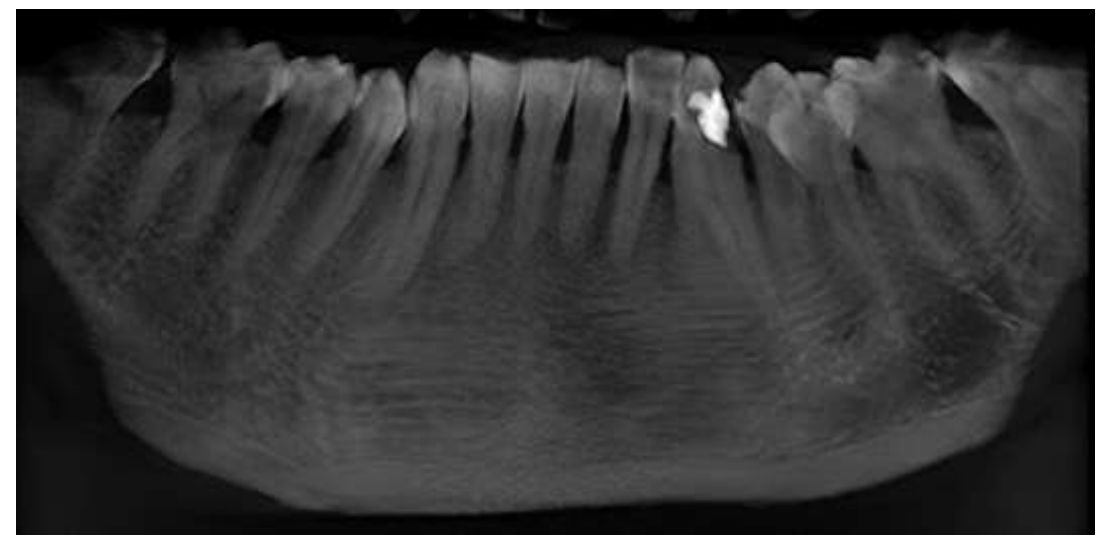

Fig3: Panoramic image 


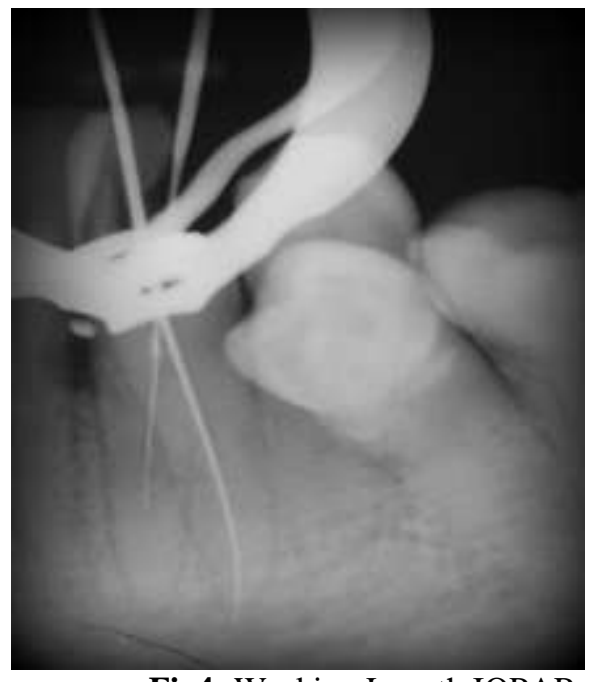

Fig4: Working Length IOPAR

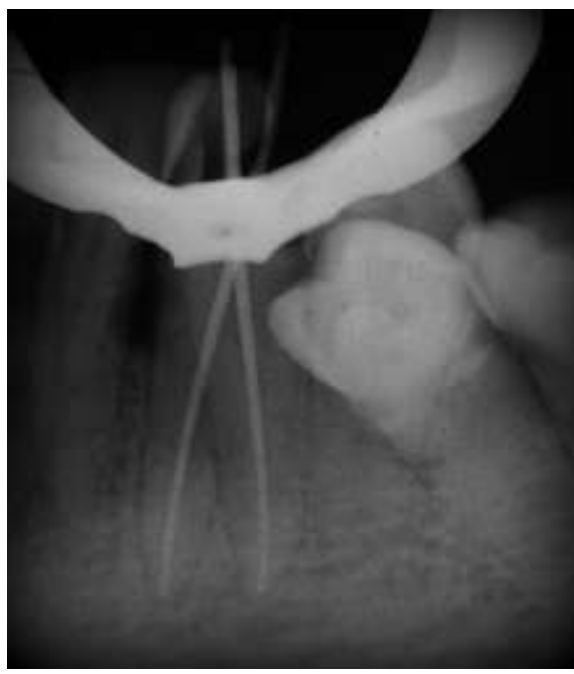

Fig5: Master Cone IOPAR

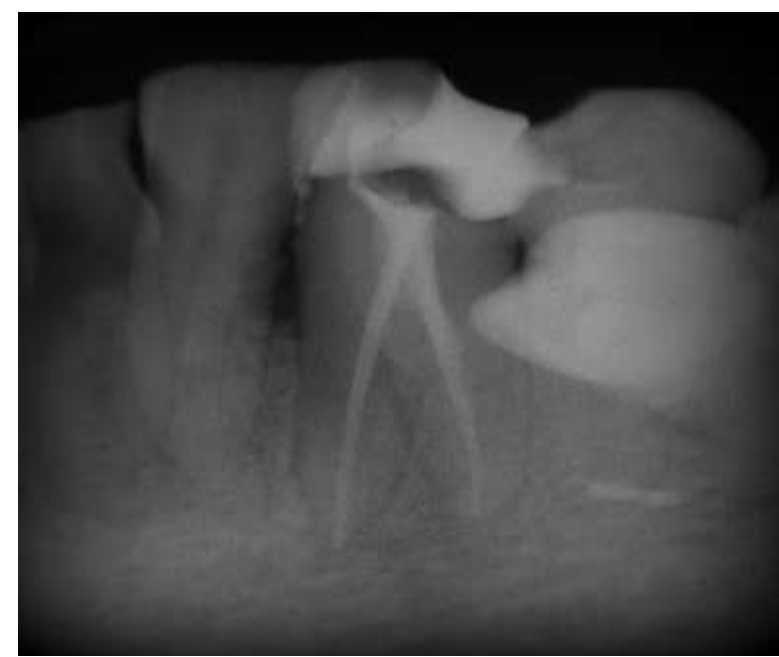

Fig6: Obturation IOPAR

\section{Conclusion}

A thorough knowledge of the internal anatomy, careful interpretation of the diagnostic radiographs with appropriate diagnostic tools and modification of the access cavity forms the important key for a successful endodontic therapy.

\section{Acknowledgements}

We extend our sincere thanks to JSD TechnoDent, Bangalore for the technical support.

\section{References}

[1]. Stewart GG. Evaluation of endodontic results DCNA 11:711,1967

[2]. Mukhaimer R, Arandi N. Endodontic Treatment of a Mandibular Canine with Two Separate Roots and Root Canals: A Case Report. Open Journal of Dentistry and Oral Medicine. 2014;2(2):38-41

[3]. Pécora JD, Sousa Neto MD, Saquy PC. Internal anatomy, direction and number of roots and size of human mandibular canines. Braz dent J. 1993 Jan;4(1):53-7.

[4]. D'Arcangelo C, Varvara G, De Fazio P. Root canal treatment in mandibular canines with two roots: a report of two cases. International Endodontic Journal. 2001 Jun 1;34(4):331-4.

[5]. Heling I, Gottlieb-Dadon I, Chandler NP. Mandibular canine with two roots and three root canals. Dental Traumatology. 1995 Dec 1;11(6):301-2

[6]. Bhardwaj A, Bhardwaj A. Mandibular Canines with Two Roots and Two Canals-A Case Report. International Journal of Dental Clinics. 2011 Dec 2;3(3).

[7]. Al-Ghananeem MM, Haddadin K, Al-Khreisat AS, Al-Weshah M, Al-Habahbeh N. The Number of Roots and Canals in the Maxillary Second Premolars in a Group of Jordanian Population. International journal of dentistry. 2014 Nov 3;2014.

[8]. Hariharavel VP, Kumar AA, Ganesh C, Annamalai S, Ramar K, Aravindhan R. Root canal treatment of mandibular second premolar with three separate roots and canals using spiral computed tomographic. Case reports in dentistry. 2014 Jul 3;2014.

[9]. Arora V, Nikhil V, Gupta J. Mandibular canine with two root canals-An unusual case report. International Journal of Stomatological Research. 2013;2(1):1-4. 
[10]. Holtzman L. Root canal treatment of a mandibular canine with three root canals. Case report. International endodontic journal. 1997 Jul 1;30(4):291-3.

[11]. Sjogren U, Figdor D, Spanberg L, Sundquist G. The antimicrobial effect of calcium hydroxide as a short term intra canal dressing. International endodontic journal. 1991 May 1;24(3):119-25.

[12]. Mohammadi Z, Shalavi S, Jafarzadeh H. Extra roots and root canals in premolar and molar teeth: Review of an endodontic challenge. J Contemp Dent Pract. 2013 Sep 1;14(5):980-6.

[13]. Eliyas S, Jalili J, Martin N. Restoration of the root canal treated tooth. British dental journal. 2015 Jan 23;218(2):53-62.

[14]. Hargreaves K M,Cohen S,Berman LH . Pathways of the pulp. 10th ed. St Louis: Mosby, 2011;785 